\title{
Clinical Value of Ultrasonography for Monitoring Angular Pregnancy in First-trimester
}

\author{
Yanhua Zhen, MD ${ }^{a}$, Xuefeng Lu, MD ${ }^{a, *}$, Chenyu Wang, MD ${ }^{a}$, Huixia Li MD ${ }^{a}$, Ji-Bin Liu, MD ${ }^{b}$ \\ ${ }^{a}$ Department of Ultrasound, The Second Affiliated Hospital of Zhengzhou University, Zhengzhou, Henan, China; ${ }^{b}$ Department of \\ Radiology, Thomas Jefferson University, Philadelphia, PA, USA \\ Received July 15, 2019; revision received August 15; accepted July August 18.
}

Objective: To explore the clinical value of ultrasonography for early detection and monitoring of angular pregnancy during the first-trimester.

\begin{abstract}
Methods: For this retrospective study, we enrolled 23 patients with asymptomatic angular pregnancy who were diagnosed by ultrasound at early pregnancy and who underwent ultrasound follow-up to determine its clinical outcome. An ultrasound unit E8 (GE Healthcare, Milwaukee, WI) associated with a 5-9 MHz convex array transvaginal probe was used to image the gestational sac by both two-dimensional and three-dimensional modes. Transvaginal sonographic observation included the location, size, and shape of the gestational sac, as well as the relationship between the gestational sac and the endometrium. When the gestational sac was located close to or at the corners of the uterus, the wall thickness of the corner was measured and documented for follow-up during the first-trimester.
\end{abstract}

Results: Angular pregnancy ( $\mathrm{n}=23$ ) was detected by first ultrasonography at 37-50 days of gestation. All patients then underwent dynamic sonographic monitoring and were closely followed every 5-10 days for 1-3 weeks. In 3 cases, ultrasound showed embryo demise at follow-up examination. Seven cases were converted to intrauterine pregnancies based on sonographic findings and carried to term. In 13 cases, ultrasonography showed the gestational sacs grew outward in the corner of the uterus where the wall was thinning and had asymmetrical corners. Based on the ultrasound results, thesepregnancies were terminated (by surgery $[n=6]$ or medication $[n=7])$.

Conclusions: Sonography is a valuable clinical tool for early detection and monitoring of angular pregnancy. Follow-up ultrasound can provide useful information for observing the transformation of angular pregnancy to guide clinical management, including whether to continue or terminate pregnancy.

Key Words: Angular pregnancy; Transvaginal ultrasound; Ultrasound monitoring; Diagnosis; Treatment; Clinical outcome

Advanced Ultrasound in Diagnosis and Therapy 2019;03:109-114

DOI: $10.37015 / A U D T .2019 .190818$

A ngular pregnancy is a rare and life-threatening condition that was first defined in 1898 by the American obstetrician Howard Kelly as implantation of the embryo just medial to the uterotubal junction, in the lateral angle of the uterine cavity and near the proximal ostium of the fallopian tube [1,2]. Angular pregnancy may cause complications during pregnancy and delivery, such as bleeding, spontaneous abortion, uterine rupture, retained placenta, placenta accreta, and hysterectomy, and given the severity of the prognosis, angular pregnancy management considerations include therapeutic abortion. However, angular pregnancy can sometimes be carried to term based on the intrauterine and intraendometrial location of the gestational sac. In

\footnotetext{
* Corresponding authors: Department of Ultrasound, The Second Affiliated Hospital of Zhengzhou University, No. 2 Jingba Road, Jingshui District, Zhengzhou 450014, China.

e-mail: luxuefeng2011@163.com
} unrestricted use, distribution and reproduction in any medium provided that the original work is properly attributed. 
this study, we aimed to demonstrate the clinical value of ultrasonography to monitor angular pregnancy and to provide the basis for its clinical treatment during this first-trimester stage.

\section{Materials and Methods}

\section{Patients}

The protocol of this study was approved by the ethics committee of Zhengzhou University, and informed consent was obtained from all participants. Twenty-three patients (average age $=38.4$ years [range 35 to 41 years]) were enrolled in this study from October 2015 to October 2018 in the Second Affiliated Hospital of Zhengzhou University. Of the 23 pregnancies, 8 resulted from assisted reproductive technology and 15 were natural pregnancy.

Inclusion criteria: (1) No uterine malformation, (2) positive human chorionic gonadotropin ( $\mathrm{HCG}>1000$ $\mathrm{mIU} / \mathrm{ml}$ ) in blood test, (3) angular pregnancy seen at the first ultrasound examination at first-trimester, (4) no pelvic pain and vaginal bleeding emergency condition, and (5) complete follow up with clinical data.

\section{Ultrasound Examination}

An ultrasound scanner (E8, GE Healthcare, Milwaukee, WI) associated with a 5-9 MHz convex array transvaginal probe was used to image the gestational sac by both two-dimensional and three-dimensional modes. All images were digitally stored on the system hard drive for documentation and later analysis. All patients with early pregnancy were examined by transvaginal ultrasound with both two-dimensional and three-dimensional modes. Ultrasound observation included vagina, cervix, uterine body, uterine cavity, and gestational sac. The ultrasound examination was focused on the location of the gestational sac, whether there was prominent uterine contour, bilateral cornual shape, cornual myometrium shape, the size of the gestational sac, and the thickness of corneal myometrium around the gestational sac, as well as the relationship between gestational sac and endometrium on the 3D rendering. The sonographic characteristics for diagnosis of angular pregnancy were: (1) the gestational sac was located at one side of the corners of the uterus, and no gestational sac could be seen in the mid-sagittal section of the uterus; (2) the endometrium was wrapped or semi-wrapped around the sac; (3) the myometrium around the sac was intact; (4) the myometrium around the corners of the uterus was not significantly shortened compared with the opposite side; and (5) the shape of the corners was changed. All the patients underwent dynamic sonographic monitoring and close follow-up every 5-10 days until a clinical decision about management was made.

\section{Results}

The first detection of angular pregnancy $(n=23)$ by ultrasonography was at 37-50 days of gestation (irregular menstruation cycle or unclear gestational weeks were measured by the first ultrasonography to correct gestational weeks). In 6 of these 23 cases, it was unclear whether the pregnancies were angular or interstitial under 2D mode ultrasound, and 2 of the pregnancies were misdiagnosed as interstitial. 3D mode ultrasound clearly showed the relationship between the gestational sac and endometrium, and the diagnosis was confirmed.

In 3 cases, ultrasound showed embryos without a consistently beating heart and embryo demise at followup exam (Table 1); the pregnancies were terminated by medication. Seven cases were converted to intrauterine pregnancies based on sonographic findings and carried to term (Fig. 1, Table 2). In 13 cases, ultrasonography demonstrated that the gestational sacs grew outward in the corner of the uterus where the wall was thinning wall and corners asymmetrical (Fig. 2). Based on these ultrasound results, these pregnancies were terminated by surgery $(n=6)$ or medication $(n=7)$ (Table 3$)$.

Table 1 The 3 cases of angular pregnancy with embryos demise

\begin{tabular}{ccccc}
\hline Case No. & Pregnancy mode & The first scanning week $\left(\mathrm{W}^{+\mathrm{D}}\right)$ & Monitoring $\left(\mathrm{W}^{+\mathrm{D}}\right)$ & Terminated week $\left(\mathrm{W}^{+\mathrm{D}}\right)$ \\
\hline 1 & Nature Conceived & $5^{+4}$ & $6^{+6} \mathrm{w}, 7^{+6} \mathrm{~W}$ & $8^{+1}$ \\
\hline 2 & Assisted Conception & $6^{+4}$ & $7^{+5} \mathrm{w}, 8^{+3} \mathrm{~W}$ & $8^{+4}$ \\
\hline 3 & Nature Conceived & $6^{+2}$ & $7^{+2} \mathrm{w}, 8 \mathrm{w}$ & $8^{+1}$ \\
\hline
\end{tabular}


Table 2 The 7 cases of angular pregnancy converted to intrauterine pregnancy

\begin{tabular}{|c|c|c|c|c|}
\hline Case No. & Pregnancy mode & $\begin{array}{l}\text { First scanning week } \\
\qquad\left(\mathrm{W}^{+\mathrm{D}}\right)\end{array}$ & $\begin{array}{l}\text { Monitoring } \\
\left(\mathrm{W}^{+\mathrm{D}}\right)\end{array}$ & $\begin{array}{l}\text { Gestational week and } \\
\text { delivery mode }\left(\mathrm{W}^{+\mathrm{D}}\right)\end{array}$ \\
\hline \multirow[t]{2}{*}{1} & Nature Conceived & $5^{+2}$ & $6^{+3} \mathrm{w}, 8 \mathrm{w}, 12 \mathrm{w}$ & $38^{+2}$ \\
\hline & & & Gestational sac extended into uterine cavity & Spontaneous delivery \\
\hline \multirow[t]{2}{*}{2} & Nature Conceived & $6^{+2}$ & $7 \mathrm{w}, 8^{+3} \mathrm{w}, 12 \mathrm{w}$ & $39^{+2}$ \\
\hline & & & Gestational sac gradually extended into uterine cavity & Cesarean section \\
\hline \multirow[t]{2}{*}{3} & Assisted Conception & $6^{+1}$ & $7 \mathrm{w}, 8 \mathrm{w}, 12 \mathrm{w}$ & $35^{+0}$ \\
\hline & & & Gestational sac gradually extended into the uterine cavity. & Cesarean section \\
\hline \multirow[t]{2}{*}{4} & Assisted Conception & $6^{+0}$ & $7 \mathrm{w}, 8 \mathrm{w}, 12 \mathrm{w}$ & $36^{+3}$ \\
\hline & & & Gestational sac gradually extended into the uterine cavity. & Cesarean section \\
\hline \multirow[t]{2}{*}{5} & Nature Conceived & $6^{+3}$ & $7 w^{+2}, 9 w, 12 w$ & $39^{+1}$ \\
\hline & & & Gestational sac gradually extended into the uterine cavity & Spontaneous delivery \\
\hline \multirow[t]{2}{*}{6} & Nature Conceived & $6^{+5}$ & $8 \mathrm{w}, 9 \mathrm{w}^{+3}, 12 \mathrm{w}$ & $39^{+5}$ \\
\hline & & & Gestational sac gradually extended into the uterine cavity & Spontaneous delivery \\
\hline \multirow[t]{2}{*}{7} & Nature Conceived & $5^{+6}$ & $7 \mathrm{w}, 8 \mathrm{w}, 12 \mathrm{w}$ & $38^{+6}$ \\
\hline & & & Gestational sac gradually extended into the uterine cavity & Cesarean section \\
\hline
\end{tabular}

Table 3 The 13 cases with angular pregnancy for termination outcome

\begin{tabular}{|c|c|c|c|c|}
\hline Case No. & Pregnancy mode & $\begin{array}{l}\text { First scanning } \\
\text { week }\left(\mathrm{W}^{+\mathrm{D}}\right)\end{array}$ & Monitoring $\left(\mathrm{W}^{+\mathrm{D}}\right)$ & $\begin{array}{l}\text { Terminated } \\
\text { week }\left(\mathrm{W}^{+\mathrm{D}}\right)\end{array}$ \\
\hline \multirow[t]{2}{*}{1} & Nature Conceived & $6^{+1}$ & $7 \mathrm{w}, 8 \mathrm{w}$ & $8^{+2}$ \\
\hline & & & Left corner convex outside and muscle layer about $3 \mathrm{~mm}$ & \\
\hline \multirow[t]{2}{*}{2} & Nature Conceived & $6^{+3}$ & $7^{+2} \mathrm{w}, 8^{+1} \mathrm{w}$ & $8^{+2}$ \\
\hline & & & Right corner convex outside and obviously asymmetrical on both sides & \\
\hline \multirow[t]{2}{*}{3} & Nature Conceived & $6^{+0}$ & $7^{+1} \mathrm{w}, 8 \mathrm{w}$ & $8^{+0}$ \\
\hline & & & Right corner convex outside and obviously asymmetrical on both sides & \\
\hline \multirow[t]{2}{*}{4} & Nature Conceived & $6^{+4}$ & $7^{+2} \mathrm{w}, 8^{+2} \mathrm{w}$ & $8^{+3}$ \\
\hline & & & Right corner convex outside and obviously asymmetrical on both sides & \\
\hline \multirow[t]{2}{*}{5} & Nature Conceived & $6^{+1}$ & $7^{+2} \mathrm{w}$ & $7^{+5}$ \\
\hline & & & Left corner convex outside and muscle layer about $4 \mathrm{~mm}$ & \\
\hline \multirow[t]{2}{*}{6} & Assisted Conception & $6^{+5}$ & $7^{+3} \mathrm{w}, 8^{+1} \mathrm{w}$ & $8^{+1}$ \\
\hline & & & Right corner convex outside and muscle layer about $4 \mathrm{~mm}$ & \\
\hline \multirow[t]{2}{*}{7} & Assisted Conception & $5^{+5}$ & $7^{+0} \mathrm{w}, 8^{+1} \mathrm{w}$ & $8^{+1}$ \\
\hline & & & Right corner convex outside and muscle layer about $4 \mathrm{~mm}$ & \\
\hline \multirow[t]{2}{*}{8} & Assisted Conception & $6^{+1}$ & $7^{+0} \mathrm{w}, 7^{+6} \mathrm{w}, 8^{+4} \mathrm{w}$ & $9^{+0}$ \\
\hline & & & Right corner convex outside and muscle layer about $4 \mathrm{~mm}$ & \\
\hline \multirow[t]{2}{*}{9} & Nature Conceived & $6^{+2}$ & $7^{+3} \mathrm{w}$ & $7^{+4}$ \\
\hline & & & Right corner convex outside and muscle layer about $4 \mathrm{~mm}$ & \\
\hline \multirow[t]{2}{*}{10} & Assisted Conception & $6^{+4}$ & $7^{+3} \mathrm{w}, 8^{+2} \mathrm{w}$ & $8^{+3}$ \\
\hline & & & Right corner convex outside and muscle layer about $3 \mathrm{~mm}$ & \\
\hline \multirow[t]{2}{*}{11} & Nature Conceived & $6^{+3}$ & $7^{+2} \mathrm{w}$ follow-up, Left corner obviously protruded and muscle layer about $5 \mathrm{~mm}$ & $8^{+1}$ \\
\hline & & & $8 w$ follow-up, Muscle layer continuously thinned by about $3 \mathrm{~mm}$. & \\
\hline \multirow[t]{2}{*}{12} & Nature Conceived & $5^{+6}$ & $7^{+0} \mathrm{~W}, 8^{+0} \mathrm{~W}$ & $8^{+2}$ \\
\hline & & & Right corner obviously protruding and muscle layer about $3 \mathrm{~mm}$ & \\
\hline \multirow[t]{2}{*}{13} & Nature Conceived & $7^{+1}$ & $8^{+1}, 9^{+0}$ & $9^{+1}$ \\
\hline & & & Left corner convex and muscle layer continuously thinned by about $3 \mathrm{~mm}$. & \\
\hline
\end{tabular}



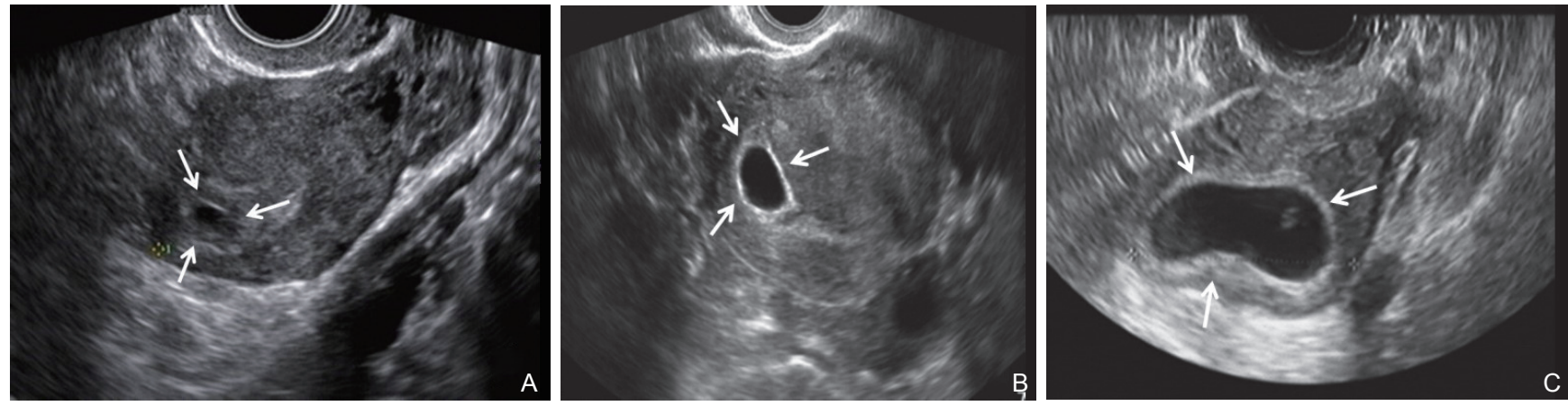

Figure 1 (A) Transvaginal ultrasound showed a gestational sac (arrows) located at the left corner side of the uterus at 5 weeks and 4 days of pregnancy; (B) The following-up ultrasound showed the sac (arrows) within the intrauterine cavity at 6 weeks and 3 days; (C) Continued intrauterine growth of the gestational sac (arrows) at 7 weeks and 3 days.
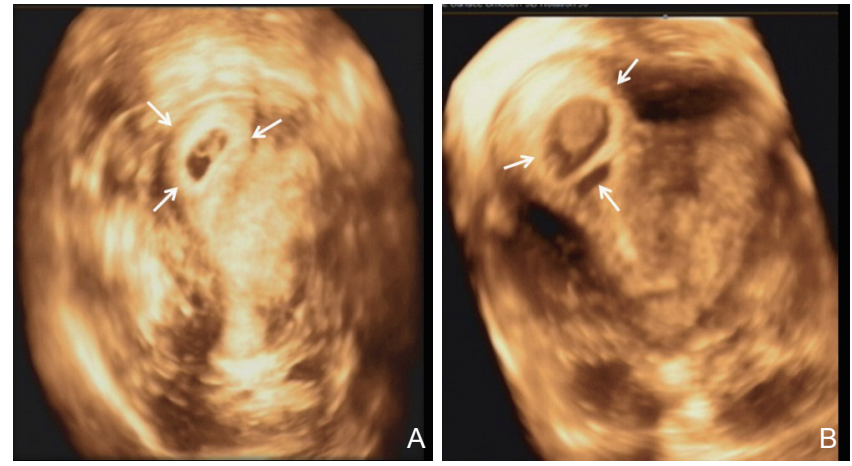

Figure 2 (A) Three-dimensional transvaginal ultrasound showed the gestational sacs (arrows) located at the corner of uterus with thinning wall and asymmetrical corners at 6 weeks and 3 days of pregnancy; (B) The follow-up ultrasound at 7 weeks and 5 days showed continue outward growth of gestational sac (arrows).

\section{Discussion}

Despite being described over a century ago, angular pregnancy remains an obscure topic. Angular pregnancy, which is located is adjacent to interstitial tubal pregnancy and is very difficult to differentiate from interstitial tubal pregnancy, is frequently misdiagnosed. The clinical differential diagnosis of angular intrauterine pregnancy from interstitial pregnancy is based on the position of the gestational enlargement of the uterus in relation to the round ligament. Because each situation has different obstetric outcomes, distinguishing between them is essential and important for clinical management. Angular pregnancies can sometimes be carried to term according to their intrauterine and intraendometrial location [3]. An angular pregnancy can sometimes be carried to term [4]. However, interstitial pregnancy is one of the most risky types of ectopic gestation, and is generally considered nonviable; it carries a mortality rate that approaches $2.5 \%$, due to the increased risk of uterine rupture and severe hemorrhage $[5,6]$. Therefore, interstitial pregnancy should be terminated immediately once confirmed.

The early clinical symptoms of angular pregnancy are atypical. The diagnostic criteria proposed by Jansen and Elliot [7] are abdominal pain, vaginal bleeding with increased uterine asymmetry, enlargement of one side of cornual uterus with lateral displacement of the circular ligament under direct laparoscopic visualization, and retention of placenta in cornual uterus.

Available evidence for angular pregnancy outcomes reveals that only a small percentage of these pregnancies result in live birth. A meta-analysis describing 39 cases of angular pregnancy reported a $38.5 \%$ chance of spontaneous abortion, $13.6 \%$ chance for uterine rupture, and a $28 \%$ live birth rate [7]. In 2018 there is an updated study with the addition of 46 subsequent cases to adjust estimation to $18 \%$ risk of spontaneous abortion and $28 \%$ risk of uterine rupture [8]. The overall live birth rate was about $25 \%$ for patients with angular pregnancy. Therefore, the management of angular pregnancy should include therapeutic abortion and observation according to the specific conditions of patients. The degree of risk burden can be chosen to wait for observation [9]. The growth trend of angular pregnancy depends on the polarity of the blastocyst, i.e. the direction of placental development and the location relationship between the gestational sac and the uterine cavity as well as the opening of the fallopian tube [10,11]. The closer the position of the gestational sac to the myometrial corner near the opening of the fallopian tube, the higher the risk of cornual rupture and massive hemorrhage with the progress of the pregnancy. On the contrary, the closer the gestational sac to the corners of the uterine cavity, the greater the possibility of inward growth of the sac. There is no absolute anatomical boundary between angular pregnancy and normal pregnancy, although the closer the distance between the implant and the uterine orifice inside the fallopian tube, the greater the possibility of visual asymmetry [7].

Diagnosis of angular pregnancy requires an imaging examination. Ultrasound is the preferred imaging method for early differential diagnosis of interstitial pregnancy and angular pregnancy. Transvaginal ultrasonography 
is an excellent method for early diagnosis of angular pregnancy because it can have better imaging resolution and display the relationship between gestational sac, uterine cavity, and fallopian tube. Since the coronal view of the uterus is difficult to present on the conventional two-dimensional imaging, transvaginal three-dimensional ultrasound can reconstruct the volume imaging into multiplanar section to show the coronal view of the uterine structure. The three-dimensional reconstruction images can clearly display the entire uterine cavity, the muscular layer of the uterine wall and the gestational sac, which can intuitively reflect the location and relationship between the implantation site of the gestational sac and the corners of the uterus. Therefore, when twodimensional transvaginal ultrasound suspects angular pregnancy, it is necessary to conduct further transvaginal three-dimensional ultrasound examination to determine the position of the gestational sac.

Ultrasound can dynamically monitor the growth direction of angular pregnancy. The clinical management of angular pregnancy can be determined by observing the shape of angular pregnancy, the symmetry of the corners on both sides, the thickness of corneal myometrium and the development of embryos by a serial of both 2D and 3D ultrasound observation. We believe that after the first diagnosis of angular pregnancy by ultrasonography, reexamination of ultrasound should be conducted every 5-7 days, which could demonstrate the growth direction and trend of the gestational sac, and then determine whether to continue pregnancy or terminate pregnancy in time. In this study, 7 cases eventually turned to intrauterine pregnancy and had live birth, while the remaining 16 cases had terminated pregnancy. The termination time of pregnancies in this study was at 8-9 weeks of the pregnancy under ultrasound monitoring.

The incidence of angular pregnancy has increased in China in recent years. Several factors may explain this increase, including comprehensive liberalization of the two-child policy in China, utilization of assisted reproductive technology, the increase in uterine cavity operations such as induced abortion, and the increase in the incidence of gynecological inflammation. These factors may all result in fertilized eggs not being implanted in the appropriate position of the uterine cavity, resulting in angular pregnancy. Because of the special position of angular pregnancy, the methods of clinical treatment vary as the gestational sac grows. The thicker the muscle layer is around the angular pregnancy, the later the rupture time could be. Even some ruptures occur in the second trimester of pregnancy [11]. Therefore, for asymptomatic early angular pregnancy patients, ultrasound can be used to dynamically observe the growth trend of the gestational sac. If the growth of the intrauterine pregnancy occurs during the followup observation, the cornual myometrial layer should not become significantly thinner and the gestational sac should be connected with the endometrium line. In addition, the corners of uterus should not have exophytic appearance. In this situation, with close observation and the patient's understanding and informed consent, the pregnancy could be carefully continued because it is possible to convert it into an intrauterine pregnancy. Once dynamic ultrasonography shows progressive exophytic corners and muscle thinning, termination of the pregnancy is necessary and critical.

Our study had several limitations. The number of cases enrolled was limited. This study did not include individuals with uterine malformations and other abnormalities since other abnormal conditions could cause angular pregnancy and may have different outcomes. The current study did not compare ultrasound to MRI. Previous studies have shown that MRI exam is useful to make an accurate diagnosis and to evaluate placental implantation and the risk of uterine rupture, but MRI is not always available and increases the cost of care $[14,15]$. Further investigation that includes some congenital uterine malformations and other abnormalities associated with angular pregnancy and that compares ultrasound results with MRI is warranted.

In general, angular pregnancy is a special abnormal pregnancy that can cause server complications such as uterine rupture. Given the serious consequences associated with angular pregnancy, it is necessary to counsel patients about potential complications and clinical management, which might include therapeutic abortion. Close monitoring and repeated ultrasound examination should be carried out to prevent complications. The clinical management should consider patient decisions [12].

In conclusion, sonography is an accurate imaging modality for evaluating the site of pregnancy implantation and a valuable clinical tool for early detection and monitoring of angular pregnancy. Followup ultrasound can provide useful information for observing the transformation of angular pregnancy and for guiding clinical management.

\section{Conflict of Interest}

The authors have no conflict of interest to declare.

\section{References}

[1] Kelly H. Operative gynecology. New York: Appleton; 1898.

[2] Ghaneie A, Grajo JR, Derr C, Kumm TR. Unusual ectopic pregnancies sonographic findings and implications for management. J Ultrasound Med 2015;34:951-62.

[3] Parker RA 3rd, Yano M, Tai AW, Friedman M, Narra VR, Menias 
CO. MR imaging findings of ectopic pregnancy: a pictorial review. Radiographics 2012;32:1445-60

[4] Doubilet PM, Benson CB, Bourne T, Blaivas M, et al. Diagnostic criteria for nonviable pregnancy early in the first trimester. $N$ Engl $J$ Med 2013;369:1443-51.

[5] Damario MA, Ectopic pregnancy, Te Linde's Operative Gynecology, 11th Ed. Lippincott Williams \& Wilkins, Philadelphia (PA) 2015; pp. 789-790.

[6] Moawad NS, Mahajan ST, Moniz MH, Taylor SE, Hurd WW. Current diagnosis and treatment of interstitial pregnancy. Am J Obstet Gynecol 2010;202:15-29.

[7] Jansen RP, Elliott PM. Angular intrauterine pregnancy. Obstet Gynecol 1981;58:167-75.

[8] Marfori CQ, Kotzen M. Angular vs. interstitial pregnancy: A case report highlighting diagnostic nuances with stark management differences. Case Rep Womens Health 2018;19:e00068.

[9] Arleo EK, DeFilippis EM. Cornual, interstitial and angular pregnancies: clarifying the term and a review of the literature. Clin
Imaging 2014;38:763-70

[10] Nadi M, Richard C, Filipuzzi L, Bergogne L, Douvier S, Sagot P. Interstitial, angular and cornual pregnancies: diagnosis, treatment and subsequent fertility. Gynecol Obstet Fertil Senol 2017;45:340-7.

[11] Veersema S, Damoiseaux A. Ruptured ectopic (cornual) pregnancy. $J$ Minim Invasive Gynecol 2009;16:666.

[12] Alanbay İ, Öztürk M, Karaşahin KE, Yenen MC. Angular pregnancy. Turk J Obstet Gynecol 2016;13:218-20..

[13] Larraín D, Marengo F, Bourdel N, Jaffeux P, Aublet-Cuvelier B, Pouly JL, et al. Proximal ectopic pregnancy: a descriptive general population-based study and results of different management options in 86 cases. Fertil Steril 2011;95:867-71.

[14] Lee MH, Im SY, Kim MK, Shin SY, Park WI. Comparison of Laparoscopic Cornual Resection and Cornuotomy for Interstitial Pregnancy. J Minim Invasive Gynecol 2017;24:397-401.

[15] Filhastre M, Dechaud H, Lesnik A, Taourel P. Interstitial pregnancy: role of MRI. Eur Radiol 2005;15:93-5. 